\title{
Clinical Outcomes and Prognostic Factors of Arthroscopic Bankart Repair for Recurrent Shoulder Instability in Soldiers: A Retrospective Single-Center Analysis
}

Ji Li

Chinese PLA General Hospital

Wei Qi

Chinese PLA General Hospital

Qiang Zhang

Chinese PLA General Hospital

Ketao Wang

Zhongshan Hospital Fudan University

Wei Li

Capital Medical University

Haoran Wang

Chinese PLA General Hospital

Zhongli Li ( $\square$ lizhongli301@126.com )

Chinese PLA General Hospital

\section{Research}

Keywords: Arthroscopy, Shoulder Dislocation, Joint Instability, Military Personnel

Posted Date: June 16th, 2020

DOI: https://doi.org/10.21203/rs.3.rs-34799/v1

License: (c) (i) This work is licensed under a Creative Commons Attribution 4.0 International License.

Read Full License 


\section{Abstract}

Background: The optimal surgical treatment of recurrent traumatic anterior shoulder instability remains a debated topic. The soldier is a special population with high-intensity military training of shoulder. The purpose of this study was to evaluate the clinical outcomes of arthroscopic Bankart repair for recurrent shoulder instability in soldiers and identify the related risk factors.

Methods: A retrospective single-center analysis was performed for soldiers with recurrent shoulder instability who underwent arthroscopic Bankart repair between 2009 and 2014. The primary outcome measure was recurrence of instability (redislocation or subluxation). Statistical analysis by binary logistic regression analysis was performed for the significance of various risk factors including sex, gender, number of preoperative dislocations (single/recurrent), the time interval between the first dislocation and the surgery (less or more than 6 months), SLAP repair and number of anchors. The functional outcomes were assessed with the visual analog scale (VAS), the American Shoulder and Elbow Surgery Score (ASES) and Rowe stability score preoperatively and at the last follow-up.

Results: A total of 95 shoulders were followed for $53.81 \pm 9.37$ months (range, 38 to 92 months). The overall postoperative recurrent instability rate was $16.84 \%$ (16 in 95). The statistical analysis showed significant differences in age $(P=0.045, O R=0.818)$, and the time interval between the first dislocation and surgery $(P=0.035, O R=5.289)$. There was a significant improvement in the mean VAS, ASES, and Rowe scores postoperatively $(P<0.05)$, but the mean VAS and Rowe scores were lower in the patients of recurrence ( $P=0.022$ and 0.034 , respectively).

Conclusions: This study suggested that surgery within 6 months of the first dislocation may be required for the young soldiers, although several repair techniques exist for anterior shoulder instability, arthroscopic Bankart repair remains a viable option.

\section{Background}

Anterior shoulder dislocation is the most common major joint dislocation(1,2), which is always complicated by recurrent instability $(3,4)$. Various biomechanical changes contribute to this recurrence. Translation of the humeral head can cause an associated avulsion of the glenoid labrum known as a Bankart lesion, which causes an increase in anterior translation in all positions of elevation (5), conferring recurrent instability(3). Surgical intervention is necessary for the patients suffering from recurrent instability of the shoulder joint following the first episode of shoulder dislocation(6).

The optimal surgical treatment of recurrent traumatic anterior shoulder instability remains a debated topic, with a number of procedures being developed over time $(7,8)$. Two of the most popular techniques are arthroscopic Bankart repair and open Lartarjet procedure. The arthroscopic Bankart technique repairs and reconstructs labrum capsule anatomically $(9,10)$, which currently the treatment of choice according to various surveys of surgeons, with $>90 \%$ of surgeons choosing the Bankart procedure as initial repair for recurrent instability(11-13). Several studies have compared these two techniques, but the rates of 
recurrence after surgeries vary widely in the literature, ranging from $0 \%$ to $30 \%$ for arthroscopic Bankart repair $(14,15)$, with a mean of $9 \%(16)$, and from $2 \%$ to $14 \%$ for the open Latarjet procedure, with a mean of $7 \%(17,18)$. In recent years, a plethora of studies have emerged to address the dislocation in athletes with highly activities $(8,19-21)$. Athletes participating in contact or collision sports are more susceptible to high-velocity impacts and repetitive positions that make their shoulders vulnerable to injury when compared with non-contact athletes(21). The arthroscopic Bankart repair has favorable results in most populations ${ }^{3-6}$, but higher failure rates have been reported in contact and collision athletes $(21,22)$. The Latarjet procedure is demonstrated to be possibly superior to a Bankart repair for athletes with professional contact sports(23). However, Latarjet procedures have been associated with a higher complication rate, such as implant failure and the development of postoperative osteoarthritis, occurring in up to $30 \%$ of cases postoperatively(24).

The soldier is a special population with high-intensity military training of shoulder, including contact and non-contact activities, any shoulder instability may significantly influence the military training and the ability to perform tasks. The purpose of this study is to evaluate the clinical outcomes of arthroscopic Bankart repair for recurrent shoulder instability in soldiers and identify the related risk factors. We hypothesized that our surgical technique of arthroscopic Bankart repair and rehabilitation protocol can provide satisfactory clinical outcomes for the soldiers' shoulder instability. To our knowledge, there is no similar studies published before.

\section{Materials And Methods}

This is a nonrandomized retrospective study. Approval was obtained from our ethics committee. Inclusion criteria included primary operations with at least a 3-year follow-up. All patients had a history of trauma to their shoulder resulting in an anterior glenohumeral dislocation confirmed on history, physical examination, and magnetic resonance imaging findings. All patients had a Bankart lesion confirmed at the time of surgery. Those with an extension of the lesion into the superior labrum (i.e., SLAP tear) were also included. Exclusion criteria included atraumatic or multidirectional instability, revision Bankart repairs, significant glenoid bone loss greater than $20 \%$ as assessed on preoperative imaging and confirmed intraoperatively, an associated rotator cuff tear, a humeral avulsion of the glenohumeral ligament, and posterior instability. The study included records of 132 soldiers received surgical interventions with shoulder anterior instability during Math 2009 and May 2014 in our department by the senior author. According to the inclusion and exclusion criteria, a total of 98 patients with 98 shoulders were further reviewed. Preoperative assessment included age, gender, and mechanism of injury, as well as the number of dislocation events prior to repair. All of them did regular physical activities for leisure or competitions, and combat activities in their military training. None had preoperative osteoarthritis injuries. All surgeries in this study were performed under arthroscopy assistance in a standardized manner by a skilled arthroscopic sports medicine trained surgeon.

\section{Surgical Technique}


General anesthesia was administered. All patients underwent an examination under anesthesia to confirm anterior instability and to exclude multidirectional instability. Patients were then placed in the lateral decubitus position, and 4-6 kg of traction was applied to the operative extremity. Preoperative antibiotic was administered within 1 hour of the start of the procedure. A posterior portal was created and used as a viewing portal. Subsequently, the glenohumeral joint was inspected and the pathology verified (Fig.1A). The anterior-superior portal was established above the subscapularis tendon as the working portal. An elevator was introduced through an anterosuperior portal to mobilize the anteroinferior labral lesion and to partially decorticate the glenoid neck. This is an important step as incomplete mobilization of the anterior labrum from the glenoid neck may result in a nonanatomic, less functional repair. A small burr was then used to create a bleeding bed of bone along the neck of the glenoid. Capsulolabral repair was performed through an anterosuperior portal using the 3.5-mm metal suture anchors (Smith \& Nephew) (Fig.1B and C). The number of devices used and the amount of capsular shifts were individualized according to the arthroscopic findings. Arthroscopic SLAP repair was simultaneously performed with suture anchors for the type II and more severe lesion, when necessary. Remplissage was used for Hill-sachs lesion when necessary. Absorbable sutures were used for skin closure, and arm of the patient was kept in a shoulder sling.

\section{Postoperative Rehabilitation}

A sling was worn for 6 weeks. In this phase, passive and gentle active assistive ROM exercises were begun, but no active external rotation, extension, or abduction was allowed. During weeks 5 to 6 , the patient worked to gradually improve ROM and initiated stretching exercises, and then the sling was removed after 6 weeks. From weeks 7 to 14, more aggressive strengthening and ROM were progressed to restore full ROM and muscular strength, and meet functional demands When full passive and active shoulder range of motion and adequate strength are achieved, the patient can progress to functional overhead activity with a full return to activity, but it is important to be aware that deficiencies in rotator cuff strength may be present. Next, enhancing muscular strength, progressing functional activities, and maintaining shoulder mobility are very important at the phase of about weeks 15 to 24 . Lastly, the return to activity phase (months 7 to 9) goals included a gradual return to military training while maintaining strength, mobility, and stability. However, in cases where a SLAP was repaired, no isolated biceps contractions were allowed until week 5 . This protocol was modified slightly for soldiers based on the athlete's goals, revision versus primary cases, and the tissue quality at the time of surgery reported in previous studies $(21,25)$.

\section{Postoperative Follow-up}

Patients were followed up in the outpatient department at 6, 12, and 24 weeks after surgeries, and every 6 months thereafter. At every follow-up, the shoulder stability status was subjectively given special attention. Frontal and lateral radiographs were obtained immediately after surgery and at every follow-up to evaluate the location of anchors and the recurrence of dislocation. At the final follow-up, radiographs 
and the clinical evaluations, including the physical examinations and scoring systems, were performed for all patients.

The primary outcome measure was recurrence of instability (redislocation or subluxation). Redislocation was verified by radiographs. Subluxation was defined as a sense of dislocation with a positive anterior apprehension test(26). Subjective assessment included the visual analog scale (VAS) for pain, the American Shoulder and Elbow Surgery Score (ASES), the Rowe Instability Score, and any postoperative complications. The VAS was based on a 1 to 10 scale, where 0 was considered no pain and 10 was considered severe pain. The ASES and Rowe score was based on a 0 to 100 scale, the higher score means the better the function. The functional scores were performed before the surgery and at the time of the final follow-up. All patient evaluations were performed by an independent observer not involved in the surgery performed. The observer is doctor working in our department and have in-depth knowledge of the disease, the surgery, and the scoring systems.

\section{Statistical Analyses}

All statistical analysis was performed using SPSS version for Windows, standard version 17.0 (SPSS, Chicago, IL). Data were expressed as percentage or mean \pm standard (SD). A priori sample size calculation was conducted based on prior studies. Using differences between presurgical and postsurgical functional scores, 50 participants would be enough to detect a significant difference with $85 \%$ power $(a=0.05)(8)$. Binary logistic regression was used to analyze the influence of each risk factor on postoperative instability. The results of regression analysis are presented as odds ratios (ORs) with accompanying $95 \%$ confidence intervals (Cls). Results were considered statistically significant when the null value (1.00) was absent from the $\mathrm{Cl}$ or $\mathrm{P}<0.05$. The paired t-test was used to compare the preoperative and postoperative scores. Independent samples t-test was applied to compare the basic quantitative data and improvement of both scores. Statistical significance was set at $\mathrm{P}<0.05$.

\section{Results}

At the final analysis, complete follow-up was achieved for 95 shoulders in 95 patients with a mean followup duration of $53.81 \pm 9.37$ months (range, 38 to 92 months). Three patient was lost to follow-up because of broken contact. There were 89 (93.68\%) males and 6 females (6.3\%), with a mean age of $23.77 \pm 4.75$ years (range, 18 to 40 years), and all of them received complete serial radiological examinations and clinical evaluations (Fig.2). Surgeries were taken place 8.73 \pm 5.26 months after the initial dislocation episode (range, 2 weeks to 4.6 years). The average number of anchors used for the Bankart repair was $5.3 \pm 2.2$. SLAP tear was present and repaired in 30 shoulders (31.58\%), including 8 of type I and 22 of type II or more severe lesion.

\section{Recurrence and Related Predictors}

Seventy-nine patients (82.11\%) reported a stable shoulder with no postoperative complications or postoperative perception of instability, and return to service and physical training. The overall 
postoperative recurrent instability rate was $16.84 \%$ (16 in 95). Among the 16 patients, 5 patients (5.26\%) experienced a redislocation following treatment with the arthroscopic Bankart procedure, one of them retired before the end of his service, two of them return to service but changed their job; $11(11.58 \%)$ reported instability or subluxation (anterior apprehension test positive) after return to training, but the shoulder did not dislocate, four of them return to service but changed their job. Other than postoperative dislocations, one patient (1.05\%) had a transient sensory brachial plexopathy due to interscalene anesthesia that resolved within 8 weeks of surgery, one patient $(1.05 \%)$ had occasional paresthesias in the arm while performing overhead activities. Among the risk factors, age at surgery, and delayed surgeries for more than 6 months from the first dislocation were significantly associated with recurrent anterior shoulder instability after the arthroscopic Bankart repairs. The average age at surgery in the recurrence patients was much younger than the part of no recurrence $(21.50 \pm 2.098$ vs $24.23 \pm 5.008$; $\mathrm{P}=0.045, \mathrm{OR}=0.818$ ). Early surgery (within 6 months from the first dislocation) was performed only in 36 of 95 patients $(37.89 \%)$. A delay of more than 6 months between the first dislocation and surgery was a significant risk factor for predicting recurrent anterior instability after the arthroscopic Bankart surgeries $(P=0.035, O R=5.289)$. Sex, times of dislocations before surgery, concomitant SLAP repair, and the number of anchors were not found to be significant risk factors for postoperative recurrent instability. The $P$ values and ORs with 95\% $\mathrm{Cl}$ for each risk factor are shown in Tables 1. 
Table 1. Univariable Binary Logistic Analysis of Risk Factors for Recurrent Instability in soldiers Who Underwent Arthroscopic Bankart Repair

\begin{tabular}{|c|c|c|c|c|c|}
\hline Risk Factors & $\begin{array}{l}\text { Recurrence }(n=16) \\
(\%)\end{array}$ & $\begin{array}{l}\text { No Recurrence } \\
(\mathrm{n}=79)(\%)\end{array}$ & $\begin{array}{l}\mathrm{P} \\
\text { Value }\end{array}$ & OR & $95 \% \mathrm{Cl}$ \\
\hline Sex & & & 0.991 & 1.014 & $\begin{array}{l}0110- \\
9.309\end{array}$ \\
\hline Male $(n=89)$ & $15(16.85)$ & 74 (84.27) & & & \\
\hline Feale $(n=6)$ & $1(16.67)$ & $5(66.67)$ & & & \\
\hline Age (mean $\pm S D)$ & $21.50 \pm 2.098$ & $24.23 \pm 5.008$ & $0.045^{\star}$ & 0.818 & $\begin{array}{l}0.672- \\
0.996\end{array}$ \\
\hline Time to surgery & & & $0.035^{\star}$ & 5.289 & $\begin{array}{l}1.126- \\
24.846\end{array}$ \\
\hline$\leq 6$ months $(n=36)$ & $2(5.56)$ & $34(94.44)$ & & & \\
\hline$>6$ months $(n=59)$ & $14(23.73)$ & 45 (76.27) & & & \\
\hline $\begin{array}{l}\text { Single/recurrent } \\
\text { dislocations }\end{array}$ & & & 0.211 & 3.810 & $\begin{array}{l}0.468- \\
31.025\end{array}$ \\
\hline Single $(n=17)$ & $1(5.88)$ & $16(94.12)$ & & & \\
\hline Multiple $(n=78)$ & $15(19.23)$ & $63(80.77)$ & & & \\
\hline SLAP repair & & & 0.707 & 1.250 & $\begin{array}{l}0.390- \\
4.010\end{array}$ \\
\hline Present $(n=30)$ & $5(16.67)$ & 25 (83.33) & & & \\
\hline Absent $(n=65)$ & $11(16.92)$ & $54(83.08)$ & & & \\
\hline No. of anchors & & & 0.391 & 2.542 & $\begin{array}{l}0.301- \\
21.444\end{array}$ \\
\hline$<3(n=11)$ & $3(27.27)$ & $8(63.64)$ & & & \\
\hline$\geq 3(n=84)$ & $13(15.48)$ & $71(73.81)$ & & & \\
\hline
\end{tabular}

\section{Functional Outcomes}

The subjective functional outcome measures used to assess functional outcomes were the VAS pain scale, ASES, and Rowe. There was a significant improvement in the mean VAS, ASES, and Rowe score postoperatively when compared with preoperatively (all of the $P$ values $<0.05$ ), but the mean scores of VAS and Rowe were significantly lower in the group with recurrence than in the group without $(P=0.022$ and 0.034, respectively). Detailed scores are summarized in Table 2. 
Table 2. Comparison of Postoperative Functional Outcomes According to the VAS, ASES and Rowe score

\begin{tabular}{|lllllll}
\hline \multicolumn{5}{c}{ Preoperative } & \multicolumn{3}{c}{ Postoperative } \\
\cline { 2 - 7 } & Recurrence & $\begin{array}{l}\text { No } \\
\text { Recurrence }\end{array}$ & $\begin{array}{l}\text { P } \\
\text { Value }\end{array}$ & Recurrence & $\begin{array}{l}\text { No } \\
\text { Recurrence }\end{array}$ & P Value \\
VAS & $5.86 \pm 1.32$ & $5.50 \pm 1.22$ & 0.666 & $2.54 \pm 1.03$ & $1.12 \pm 0.43$ & $0.022^{\#}$ \\
\hline ASES & $47.2 \pm 7.22$ & $48.00 \pm 10.82$ & 0.894 & $82.88 \pm 6.61$ & $87.64 \pm 6.72$ & 0.292 \\
\hline Rowe & $38.2 \pm 9.63$ & $34.6 \pm 5.32$ & 0.485 & $76.76 \pm 5.60$ & $85.18 \pm 4.75$ & $0.034^{\#}$ \\
\hline
\end{tabular}

NOTE. Data are presented as mean \pm standard deviation. "Statistically significant different

\section{Discussion}

Arthroscopic Bankart repair and the open Latarjet bone block procedure are widely considered mainstays for surgical treatment of recurrent anterior shoulder instability ${ }^{(27)}$. Recently, the Latarjet technique has attracted enormous interests, and more and more surgeons chose it for the treatment of recurrent anterior shoulder instability. However, the choice between these two procedures was seemed to depend mainly on the surgeons' preference or training rather than on published evidence ${ }^{(27)}$. Some studies analyzed also demonstrated the cultural bias noted in the selection of surgical technique, and the heterogeneity of the study population in terms of the level of playing and sport played also make comparison difficult(25). In our clinical practice, we may choose the Latarjet procedure for the glenoid bone loss $>20 \%$; arthroscopic Bankart repair was used when glenoid bone loss is less than $20 \%$. Studies indicated that Bankart repair may be inferior to Latarjet procedure for athletes with professional contact sports $(23)^{28,29}$, however, according to our experience, arthroscopic Bankart repair may be remain an excellent treatment option, even for the active population.

Currently, there is no consensus on the type of sport and the risk of recurrence $(28,29)$. Several publications reported increased risk of recurrence in contact athletes $(20,21,30)$, whereas others did not find a significant difference(31-33). Unfortunately, there is high variability in the classification of different sports that makes comparison difficult, and none of the present classifications was suitable for this study. The soldier is a special population with high-intensity military training of shoulder, any shoulder instability may influence the military training and the ability to perform military duties(34). In the present work, we performed the arthroscopy Bankart repair for soldiers. After surgeries, the overall recurrence rate at a mean follow-up of $53.81 \pm 9.37$ months was $16.84 \%$ ( 16 of 95 shoulders). Five of the 16 patients reported only a single postoperative dislocation. Most of these did not require reduction by a physician, and none reported any further dislocation. According to the recurrence rate of arthroscopy Bankart surgery of $4 \%$ to $19 \%$ in the previous studies $(8,16,31)$, our failure rates are relatively high. However, in this study, recurrence was defined as redislocation and subluxation in contrast to other studies, which only considered redislocation. We believe that subjectively reported subluxation or apprehension alone without clinically confirmed signs of instability were not considered as failure. Furthermore, the soldier 
was a relatively young population with high activities, while in previous studies, age was the parameter most frequently associated with the risk of recurrence. Therefore, we argue that the definition of recurrence and relatively young age of the patients included in this study contributed to the relatively high recurrence instability rate. Additionally, significant improvements in ASES, Rowe, and VAS scores were noted, which was similar to Ide et al. reported on 55 patients with significant improvement in Rowe scores following arthroscopic suture anchor repair(32). However, results showed that patients who had no recurrence reported less pain when compared with patients who had recurrence.

Age has been the most frequently associated with an increased risk of dislocation after arthroscopic Bankart repair $(8,35)$. Kandziora et al. found a significant negative correlation between postoperative dislocation and the age at the time of surgery(36). In this study, our patients were relatively young, and we found that the average age of recurrence patient was significantly lower than that of patients who had no instability, which mean that the lower age was the risk factor of recurrence after the arthroscopic Bankart surgery for soldiers. We inferred this is related to relatively low musculoskeletal maturity and lacking training skills in the young soldiers. Furthermore, Porcellini et al. described that patients who were surgically managed more than 6 months after the first dislocation were 2.6 times more likely to have a redislocation in comparison with those who were managed within 6 months(37). In this study, we found that the soldiers who were surgically managed more than 6 months after the first dislocation were 5.289 times more likely to have an instability than those who were managed within 6 months $(P=0.035)$, which indicated that the time to surgery was also the risk factor of recurrence after the arthroscopic Bankart repair for soldiers. This may be attributed to the greater damage to the capsule ligamentous structures during the military training. The significantly higher prevalence of glenohumeral arthropathy in patients who underwent surgery after multiple dislocations or experienced surgical delay after the first dislocation leads us to recommend performing surgery within 6 months of the first dislocation, particularly in young and active patients, similarly to the recommendations of previous studies $(36,38)$. However, we did not found a difference in outcomes in patients undergoing single or recurrent dislocations before the surgeries, although Kandziora et al. found that the frequency of postoperative recurrent dislocation increased significantly with the number of preoperative dislocations(36). This result may be related to the smaller number of patients with single dislocation. Using less than 3 anchors has also been demonstrated to negatively affect outcomes(39). In this study, all recurrences in our study occurred in patients received 3 or more anchors. Perhaps the majority of our patients received at least 3 or more anchors, which likely diluted the effect of the number of anchors on recurrence and return to training rates. However, we believe the high number of anchors used likely contributed to a reduction in recurrence rates. Additionally, in this study, we had only 6 female patients, meaning that the study was almost certainly underpowered to detect differences in the instability between males and females.

Cho et al. report that $11 \%$ to $57 \%$ of the patients with recurrent dislocation had combined Bankart and SLAP lesions (14), in the present study, the rate was $31.58 \%$ (30/92). SLAP lesions can lead to increased glenohumeral translations, resulting in anterior shoulder instability (40),thus combined Bankart/SLAP repair is adopted by most surgeons (41). In this study, we performed arthroscopic combined repair for all the 30 patients. For the type I, we chose debridement for the SLAP lesion; repair was performed with 
anchors for the type II and more severe SLAP lesion when necessary. Arthroscopic repair using suture anchors have produced favorable clinical results in terms of pain reduction and shoulder function improvement (14). The management for the combined Bankart/SLAP lesions were still controversial in some recent literature. According to Feng $S$ et al, patients who underwent arthroscopic combined repair was proven to be a viable option for anterior shoulder instability with a low recurrence rate, favorable functional scores, and no significant restriction in ROM (42). Hantes et al. found no difference in outcomes between arthroscopic Bankart repairs with and without concomitant SLAP repairs (43), Carreira and colleagues reported improved outcomes in patients who did not undergo SLAP repair(33). In the present study, the recurrence rate in the patients who received combined Bankart/SLAP repair was 5.43\% (5/92), which was lower compared with isolated Bankart repair according to existing literature $(44,45)$. Additionally, we also did not identify a difference in outcomes in patients undergoing concomitant SLAP repair after the analysis, suggesting that properly performed combined repair does not lead to a higher risk of recurrent anterior shoulder instability than isolated repair. Consistent with previous studies (46) (47), this result indicates that appropriate repair of labral lesions may be the most critical factor to avoid recurrent dislocations after arthroscopic management of anterior shoulder instability.

The data presented in this study added useful information to the existing body of literature. Unlike other studies, we included a special population who are relatively young and with high-intensity activities for about 4.5 years' follow-up. Latarjet has been used with good results for American Midshipmen, but this procedure is no longer used in the first intention in the US, and the Bankart procedure is used in the first intention if there is no important bone loss(48). The aim of this study was not to establish the ideal repair method, arthroscopic Bankart repairs remains a viable option even for the highly active patient population.

\section{Limitation}

The current study has several limitations. Firstly, it is a nonrandomized retrospective series rather than a prospective one, preoperative outcome questionnaires were not available for review, which makes recall bias problematic. However, the postoperative values reported here are in agreement with other published data $(31,33)$. This is a single-center study and has a small sample size $(n=95)$, which resulted in the limited significance of the results. The follow-up period is not long enough to evaluate the long-term clinical efficacy. Future prospective randomized studies with longer follow-up should be performed. Secondly, the small number of recurrences in the group results in small differences in subgroup comparisons. This in turn means the study may be underpowered to truly detect some of the subgroup differences. However, the results presented are still valuable as this represents a unique population. This study still proved the effectiveness of the arthroscopic Bankart technique. Thirdly, the extraordinary demands on the shoulder joint during military training may be a special area in which there is still no an effective and objective way to evaluate. The different training demands among different army services should also not be ignored.

\section{Conclusion}


This study suggested that surgery within 6 months of the first dislocation may be required for the young soldiers, although several repair techniques exist for anterior shoulder instability, arthroscopic Bankart repair remains a viable option.

\section{Declarations}

\section{Ethics approval and consent to participate}

This is a retrospective clinical study approved by the Ethical Review Committee, General Hospital of PLA.

\section{Consent for publication}

All presentations of case reports have consent for publication.

\section{Availability of data and materials}

The datasets used and/or analysed during the current study are available from the corresponding author on reasonable request.

\section{Competing interests}

All the authors declare that they have no competing interests.

\section{Funding}

None

\section{Authors' contributions}

$\mathrm{JL}$ carried out the data analysis and drafted the manuscript. ZL conceived of the study, participated in its design and coordination, and helped to draft the manuscript. WQ and QZ participated in the design of the study and performed the statistical analysis. KT, WL and HR helped to collect data and performed the statistical analysis. All authors have been actively involved in the drafting and critical revision of the manuscript, and each provided final approval of the version to be published.

\section{Acknowledgements}

The authors thank Zhidong Zhao and Yuxing Wang who contributed toward the analysis and interpretation of the data involved in drafting the manuscript. The authors are also grateful to American Journal Experts for language help.

\section{References}

1. Simonet WT, Melton LJ, 3rd, Cofield RH, Ilstrup DM. Incidence of anterior shoulder dislocation in Olmsted County, Minnesota. Clinical orthopaedics and related research. 1984(186):186-91. 
2. Zacchilli MA, Owens BD. Epidemiology of shoulder dislocations presenting to emergency departments in the United States. The Journal of bone and joint surgery American volume. 2010;92(3):542-9.

3. Olds $\mathrm{M}$, Ellis R, Donaldson $\mathrm{K}$, Parmar P, Kersten P. Risk factors which predispose first-time traumatic anterior shoulder dislocations to recurrent instability in adults: a systematic review and metaanalysis. British journal of sports medicine. 2015;49(14):913-22.

4. Robinson $\mathrm{CM}$, Howes J, Murdoch $\mathrm{H}$, Will E, Graham C. Functional outcome and risk of recurrent instability after primary traumatic anterior shoulder dislocation in young patients. The Journal of bone and joint surgery American volume. 2006;88(11):2326-36.

5. Speer KP, Deng X, Borrero S, Torzilli PA, Altchek DA, Warren RF. Biomechanical evaluation of a simulated Bankart lesion. The Journal of bone and joint surgery American volume. 1994;76(12):1819-26.

6. Pulavarti RS, Symes TH, Rangan A. Surgical interventions for anterior shoulder instability in adults. The Cochrane database of systematic reviews. 2009(4):CD005077.

7. Millett PJ, Clavert P, Warner JJ. Open operative treatment for anterior shoulder instability: when and why? The Journal of bone and joint surgery American volume. 2005;87(2):419-32.

8. Voos JE, Livermore RW, Feeley BT, Altchek DW, Williams RJ, Warren RF, et al. Prospective evaluation of arthroscopic bankart repairs for anterior instability. The American journal of sports medicine. 2010;38(2):302-7.

9. Bach BR, Jr., O'Brien SJ, Warren RF, Leighton M. An unusual neurological complication of the Bristow procedure. A case report. The Journal of bone and joint surgery American volume. 1988;70(3):45860.

10. Kim DW, Kim CK, Jung SW. An arthroscopic pleated capsular shift for recurrent anterior dislocation of the shoulder. Knee surgery, sports traumatology, arthroscopy : official journal of the ESSKA. 2012;20(12):2579-84.

11. Berendes TD, Pilot P, Nagels J, Vochteloo AJ, Nelissen RG. Survey on the management of acute firsttime anterior shoulder dislocation amongst Dutch public hospitals. Archives of orthopaedic and trauma surgery. 2015;135(4):447-54.

12. Boileau P, Richou J, Lisai A, Chuinard C, Bicknell RT. The role of arthroscopy in revision of failed open anterior stabilization of the shoulder. Arthroscopy : the journal of arthroscopic \& related surgery : official publication of the Arthroscopy Association of North America and the International Arthroscopy Association. 2009;25(10):1075-84.

13. Chong $M$, Karataglis $D$, Learmonth $D$. Survey of the management of acute traumatic first-time anterior shoulder dislocation among trauma clinicians in the UK. Annals of the Royal College of Surgeons of England. 2006;88(5):454-8.

14. Cho HL, Lee CK, Hwang TH, Suh KT, Park JW. Arthroscopic repair of combined Bankart and SLAP lesions: operative techniques and clinical results. Clin Orthop Surg. 2010;2(1):39-46. 
15. Koss S, Richmond JC, Woodward JS, Jr. Two- to five-year followup of arthroscopic Bankart reconstruction using a suture anchor technique. The American journal of sports medicine. 1997;25(6):809-12.

16. Hobby J, Griffin D, Dunbar M, Boileau P. Is arthroscopic surgery for stabilisation of chronic shoulder instability as effective as open surgery? A systematic review and meta-analysis of 62 studies including 3044 arthroscopic operations. The Journal of bone and joint surgery British volume. 2007;89(9):1188-96.

17. Burkhart SS, De Beer JF, Barth JR, Cresswell T, Roberts C, Richards DP. Results of modified Latarjet reconstruction in patients with anteroinferior instability and significant bone loss. Arthroscopy : the journal of arthroscopic \& related surgery : official publication of the Arthroscopy Association of North America and the International Arthroscopy Association. 2007;23(10):1033-41.

18. Allain J, Goutallier D, Glorion C. Long-term results of the Latarjet procedure for the treatment of anterior instability of the shoulder. The Journal of bone and joint surgery American volume. 1998;80(6):841-52.

19. Hubbell JD, Ahmad S, Bezenoff LS, Fond J, Pettrone FA. Comparison of shoulder stabilization using arthroscopic transglenoid sutures versus open capsulolabral repairs: a 5-year minimum follow-up. The American journal of sports medicine. 2004;32(3):650-4.

20. Castagna A, Delle Rose G, Borroni M, Cillis BD, Conti M, Garofalo R, et al. Arthroscopic stabilization of the shoulder in adolescent athletes participating in overhead or contact sports. Arthroscopy : the journal of arthroscopic \& related surgery : official publication of the Arthroscopy Association of North America and the International Arthroscopy Association. 2012;28(3):309-15.

21. Yamamoto N, Kijima H, Nagamoto H, Kurokawa D, Takahashi H, Sano H, et al. Outcome of Bankart repair in contact versus non-contact athletes. Orthopaedics \& traumatology, surgery \& research : OTSR. 2015;101(4):415-9.

22. Rouleau DM, Hebert-Davies J, Djahangiri A, Godbout V, Pelet S, Balg F. Validation of the instability shoulder index score in a multicenter reliability study in 114 consecutive cases. The American journal of sports medicine. 2013;41(2):278-82.

23. An VV, Sivakumar BS, Phan K, Trantalis J. A systematic review and meta-analysis of clinical and patient-reported outcomes following two procedures for recurrent traumatic anterior instability of the shoulder: Latarjet procedure vs. Bankart repair. Journal of shoulder and elbow surgery. 2016;25(5):853-63.

24. Griesser MJ, Harris JD, McCoy BW, Hussain WM, Jones MH, Bishop JY, et al. Complications and reoperations after Bristow-Latarjet shoulder stabilization: a systematic review. Journal of shoulder and elbow surgery. 2013;22(2):286-92.

25. Milchteim C, Tucker SA, Nye DD, Lamour RJ, Liu W, Andrews JR, et al. Outcomes of Bankart Repairs Using Modern Arthroscopic Technique in an Athletic Population. Arthroscopy : the journal of arthroscopic \& related surgery : official publication of the Arthroscopy Association of North America and the International Arthroscopy Association. 2016;32(7):1263-70. 
26. Takeda H, Watarai K, Ganev GG, Oguro K, Higashi A, Tateishi A. Modified Bankart procedure for recurrent anterior dislocation and subluxation of the shoulder in athletes. International orthopaedics. 1998;22(6):361-5.

27. Bessiere C, Trojani C, Carles M, Mehta SS, Boileau P. The open latarjet procedure is more reliable in terms of shoulder stability than arthroscopic bankart repair. Clinical orthopaedics and related research. 2014;472(8):2345-51.

28. Balg F, Boileau P. The instability severity index score. A simple pre-operative score to select patients for arthroscopic or open shoulder stabilisation. The Journal of bone and joint surgery British volume. 2007;89(11):1470-7.

29. Privitera DM, Sinz NJ, Miller LR, Siegel EJ, Solberg MJ, Daniels SD, et al. Clinical Outcomes Following the Latarjet Procedure in Contact and Collision Athletes. The Journal of bone and joint surgery American volume. 2018;100(6):459-65.

30. Cho NS, Lubis AM, Ha JH, Rhee YG. Clinical results of arthroscopic bankart repair with knot-tying and knotless suture anchors. Arthroscopy : the journal of arthroscopic \& related surgery : official publication of the Arthroscopy Association of North America and the International Arthroscopy Association. 2006;22(12):1276-82.

31. Thal R, Nofziger M, Bridges M, Kim JJ. Arthroscopic Bankart repair using Knotless or BioKnotless suture anchors: 2- to 7-year results. Arthroscopy : the journal of arthroscopic \& related surgery : official publication of the Arthroscopy Association of North America and the International Arthroscopy Association. 2007;23(4):367-75.

32. Ide J, Maeda S, Takagi K. Arthroscopic Bankart repair using suture anchors in athletes: patient selection and postoperative sports activity. The American journal of sports medicine. 2004;32(8):1899-905.

33. Carreira DS, Mazzocca AD, Oryhon J, Brown FM, Hayden JK, Romeo AA. A prospective outcome evaluation of arthroscopic Bankart repairs: minimum 2-year follow-up. The American journal of sports medicine. 2006;34(5):771-7.

34. de l'Escalopier N, Barbier O, Demoures T, Ollat D, Versier G. Long-Term Results of a Monocentric Series of Soldiers After Latarjet Procedure for Anterior Shoulder Instability. Implications for the Assessment of Soldiers' Medical Ability. Military medicine. 2018;183(1-2):e134-e7.

35. Ahmed I, Ashton F, Robinson CM. Arthroscopic Bankart repair and capsular shift for recurrent anterior shoulder instability: functional outcomes and identification of risk factors for recurrence. The Journal of bone and joint surgery American volume. 2012;94(14):1308-15.

36. Kandziora F, J?Ger A, Bischof F, Herresthal J, Starker M, Mittlmeier T. Arthroscopic labrum refixation for post-traumatic anterior shoulder instability: Suture anchor versus transglenoid fixation technique. Arthroscopy-the Journal of Arthroscopic \& Related Surgery. 2000;16(4):359-66.

37. Porcellini G, Campi F, Pegreffi F, Castagna A, Paladini P. Predisposing factors for recurrent shoulder dislocation after arthroscopic treatment. The Journal of bone and joint surgery American volume. 2009;91(11):2537-42. 
38. Imhoff AB, Patrick A, Thomas T, Christoph R, Christoph B, Maximilian H, et al. Arthroscopic repair of anterior-inferior glenohumeral instability using a portal at the 5:30-o'clock position: analysis of the effects of age, fixation method, and concomitant shoulder injury on surgical outcomes. American Journal of Sports Medicine. 2010;38(9):1795-803.

39. Lee SH, Lim KH, Kim JW. Risk Factors for Recurrence of Anterior-Inferior Instability of the Shoulder After Arthroscopic Bankart Repair in Patients Younger Than 30 Years. Arthroscopy : the journal of arthroscopic \& related surgery : official publication of the Arthroscopy Association of North America and the International Arthroscopy Association. 2018;34(9):2530-6.

40. Patzer T, Habermeyer P, Hurschler C, Bobrowitsch E, Wellmann M, Kircher J, et al. The influence of superior labrum anterior to posterior (SLAP) repair on restoring baseline glenohumeral translation and increased biceps loading after simulated SLAP tear and the effectiveness of SLAP repair after long head of biceps tenotomy. J Shoulder Elbow Surg. 2012;21(11):1580-7.

41. Kim DS, Yi CH, Yoon YS. Arthroscopic repair for combined Bankart and superior labral anterior posterior lesions: a comparative study between primary and recurrent anterior dislocation in the shoulder. Int Orthop. 2011;35(8):1187-95.

42. Feng S, Song Y, Li H, Chen J, Chen J, Chen S. Outcomes for Arthroscopic Repair of Combined Bankart/SLAP Lesions in the Treatment of Anterior Shoulder Instability: A Systematic Review and Meta-analysis. Orthop J Sports Med. 2019;7(10):2325967119877804.

43. Hantes ME, Venouziou Al, Liantsis AK, Dailiana ZH, Malizos KN. Arthroscopic repair for chronic anterior shoulder instability: a comparative study between patients with Bankart lesions and patients with combined Bankart and superior labral anterior posterior lesions. The American journal of sports medicine. 2009;37(6):1093-8.

44. Bessière $C$, Trojani C, Carles M, Mehta SS, Boileau P. The open latarjet procedure is more reliable in terms of shoulder stability than arthroscopic bankart repair. Clin Orthop Relat Res. 2014;472(8):234551.

45. Thomazeau H, Courage O, Barth J, Pélégri C, Charousset $C$, Lespagnol F, et al. Can we improve the indication for Bankart arthroscopic repair? A preliminary clinical study using the ISIS score. Orthop Traumatol Surg Res. 2010;96(8 Suppl):S77-83.

46. Kim DS, Yi CH, Kwon KY, Oh JR. Relationship between the extent of labral lesions and the frequency of glenohumeral dislocation in shoulder instability. Knee Surg Sports Traumatol Arthrosc. 2013;21(2):430-7.

47. Durban CM, Kim JK, Kim SH, Oh JH. Anterior Shoulder Instability with Concomitant Superior Labrum from Anterior to Posterior (SLAP) Lesion Compared to Anterior Instability without SLAP Lesion. Clin Orthop Surg. 2016;8(2):168-74.

48. Schroder DT, Provencher MT, Mologne TS, Muldoon MP, Cox JS. The modified Bristow procedure for anterior shoulder instability: 26-year outcomes in Naval Academy midshipmen. The American journal of sports medicine. 2006;34(5):778-86. 


\section{Figures}
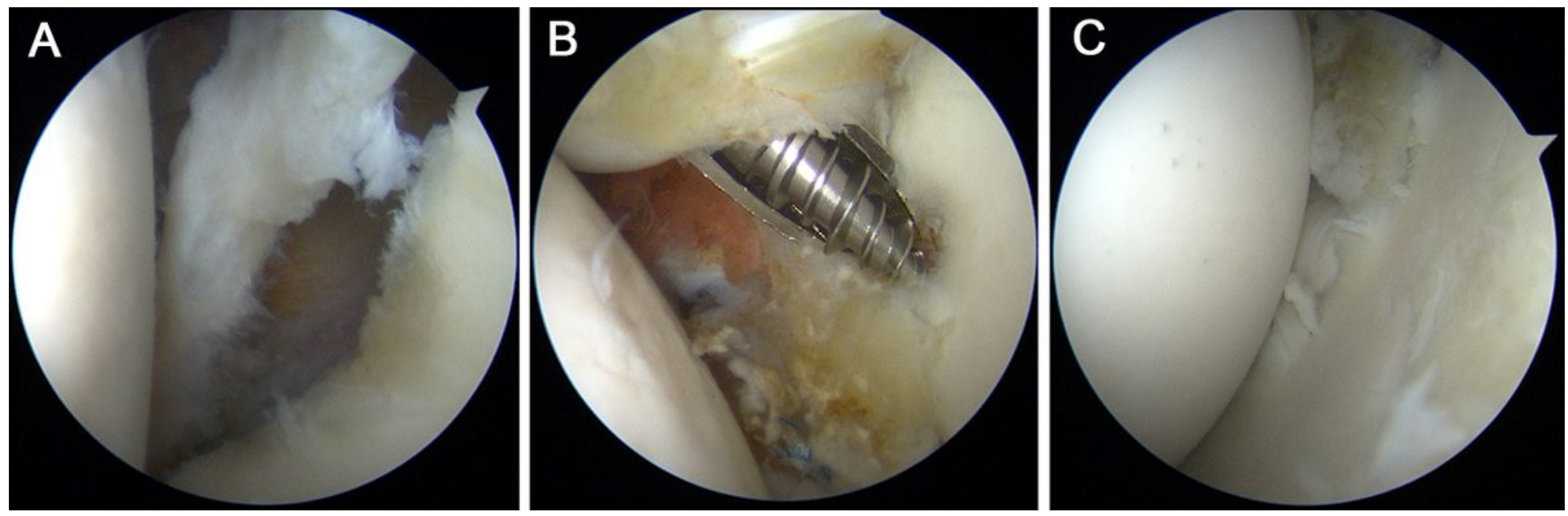

Figure 1

Arthroscopic pictures of left shoulder of a 21 male year-old soldier: (A) The glenoid labium was separated from the glenoid rim; (B) Capsulolabral repair was performed through an anterosuperior portal using the 3.5-mm metal suture anchors; (C) The final repair was done using 3 anchors, and the ligamentous and capsulolabral were reset.
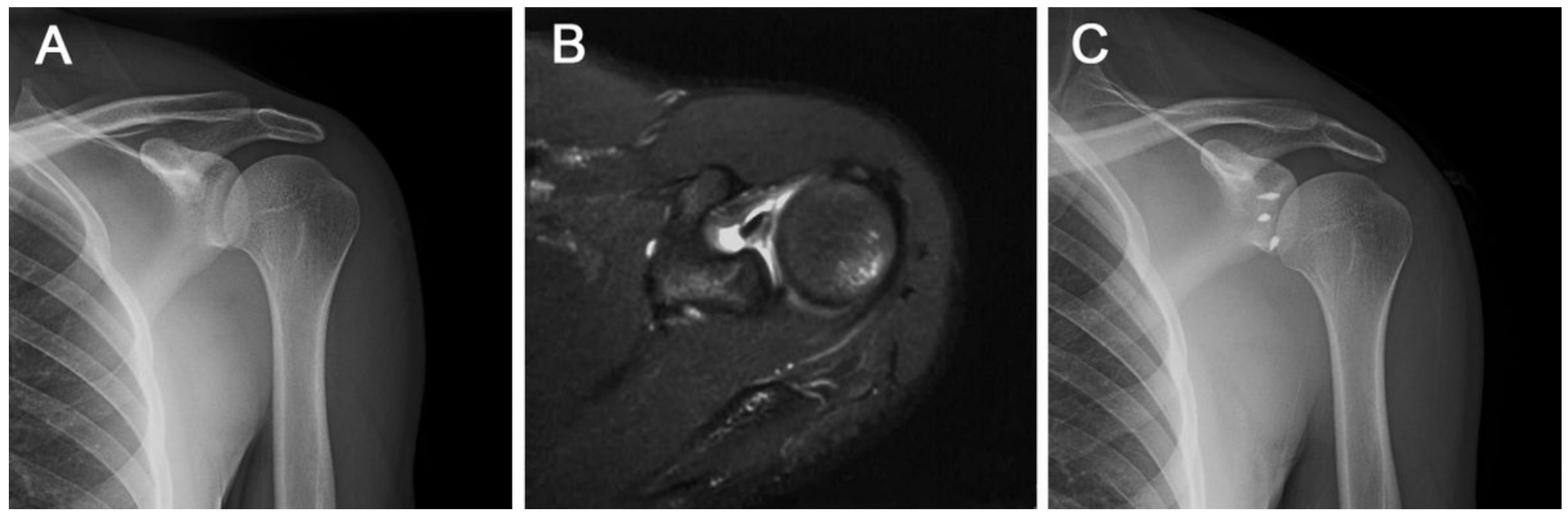

Figure 2

Images of left shoulder of a 20 year-old male soldier: (A) Preoperative radiograph; (B) Preoperative MRI image showed obvious Bankart lesion; (C) Postoperative radiograph showed three metal anchors.

\section{Supplementary Files}

This is a list of supplementary files associated with this preprint. Click to download.

- Titlepage.docx 
- Titlepage.docx

Page 17/17 